\title{
Insights into Engine Knock: Comparison of Knock Metrics across Ranges of Intake Temperature and Pressure in the CFR Engine
}

Rockstroh, Toby; Kolodziej, Christopher P.; Jespersen, Mads Carsten; Goldsborough, S. Scott; Wallner, Thomas

Published in:

S A E International Journal of Fuels and Lubricants

Link to article, DOI:

$10.4271 / 2018-01-0210$

Publication date:

2018

Document Version

Peer reviewed version

Link back to DTU Orbit

Citation (APA):

Rockstroh, T., Kolodziej, C. P., Jespersen, M. C., Goldsborough, S. S., \& Wallner, T. (2018). Insights into Engine Knock: Comparison of Knock Metrics across Ranges of Intake Temperature and Pressure in the CFR Engine. $S$ A E International Journal of Fuels and Lubricants, 2018-(4), 545-561. https://doi.org/10.4271/2018-01-0210

\section{General rights}

Copyright and moral rights for the publications made accessible in the public portal are retained by the authors and/or other copyright owners and it is a condition of accessing publications that users recognise and abide by the legal requirements associated with these rights.

- Users may download and print one copy of any publication from the public portal for the purpose of private study or research.

- You may not further distribute the material or use it for any profit-making activity or commercial gain

- You may freely distribute the URL identifying the publication in the public portal 


\section{Insights into Engine Knock: Comparison of Knock Metrics across Ranges of Intake Temperature and Pressure in the CFR Engine}

Author, co-author (Do NOT enter this information. It will be pulled from participant tab in

\begin{abstract}
Of late there has been a resurgence in studies investigating parameters that quantify combustion knock in both standardized platforms and modern spark-ignition engines. However, it is still unclear how metrics such as knock (octane) rating, knock onset and knock intensity are related, and how fuels behave according to these metrics across a range of conditions.
\end{abstract}

As part of an ongoing study, the air supply system of a standard Cooperative Fuel Research (CFR) F1/F2 engine was modified to allow mild levels of intake air boosting while staying true to its intended purpose of being the standard device for ASTM-specified knock rating, or octane number tests. For instance, the carburation system and intake air heating manifold are not altered, but the engine was equipped with cylinder pressure transducers to enable both, logging of the standard knockmeter read-out, as well as state-of-theart indicated data.

For this study, the engine was operated using primary reference fuel 90 (PRF90) at $600 \mathrm{rpm}$, first following the procedures of the ASTM D2699 research octane number test protocol in order to define the geometric compression ratio set point for standard knock number. Thereafter, compression ratio sweeps were conducted at intake temperatures ranging from 30 to $150{ }^{\circ} \mathrm{C}$ and intake air boost extending from 0 to 0.3 bar above ambient. The resulting operating map provided a broad envelope of compressed in-cylinder conditions relevant to modern spark ignition engines.

Detailed analysis of the indicated data highlighted a poor correlation between established knock intensity metrics and the knockmeter reading, which is used to characterize a fuel's octane number. It was further found that the autoignition characteristics of PRF90 could be perturbed by means of intake air boosting and heating without being captured by the knockmeter reading.

\section{Introduction}

Engine knock is the noise transmitted through the engine block due to pressure waves that resonate within the combustion chamber. These are initiated by auto-ignition of some fraction of the end gas ahead of the propagating flame front. It is understood that the auto-ignition originates from so-called "hot spots" in the end gas, that are caused by non-uniformities in temperature or fuel distribution [1, 2]. The rapid release of chemical energy from that section of the end gas and the concurrent gas dynamics, occur very rapidly, resulting in a high local pressure gradient which can lead to very strong pressure waves. These pressure discontinuities can force auto-ignition of portions of the remaining end gas, where coherence can manifest in the formation of a detonation front [2]. Apart from being an annoyance to Page 1 of 17 the operator, these pressure discontinuities can lead to catastrophic structural damage to engine components.

The constraints imposed by knock on spark ignition engine operation have been appreciated, to some degree, since automobiles gained in popularity during the early 1920's, as is evident by an array of early literature on the subject of engine knock or "detonation" by Ricardo [3], Midgley and Boyd [4] as well as others [5, 6, 7, 8]. Faced with the challenge of defining a knock specification, or ranking for automotive fuels, pioneering work by a group of oil and automotive companies led to the development of the Cooperative Fuel Research (CFR) engine and the research octane number (RON) specification in the 1930's [9]. However, it was soon discovered that the combustion chamber surface temperatures of the CFR engine during the RON test were not representative of automobile engines at the time. While meeting the RON specification, some samples of gasoline were found to behave differently in the field. The RON method was modified to become the motor octane number (MON) method, by increasing the engine speed as well as specifying a mixture temperature in order to obtain representative combustion chamber surface temperatures of engines at the time [9].

Due to ongoing development in the engine and fuel arena, it is therefore of little surprise that the RON and MON method no longer encapsulate the operating regime of modern engines [10, 11, 12], and this has motivated the development and utilization of other fuel ratings, such as the octane index, which was formulated by Kalghatgi as a practical means to correlate RON and MON to Knock Limited Spark Advance (KLSA) in modern engines [11, 13, 14]. Iterations of the octane knock index have also been applied to predict the performance of gasoline-type fuels in compression ignition engines $[15,16,17,18,14]$, where the onset of auto-ignition arguably plays an even greater role than in SI engines in operational characteristics. It is interesting to note that various improvements to the octane rating system, such as including weighting factors for fuel composition, as done by Lacey et al. [18], were reportedly considered in the 1960's [9].

The CFR engine remains the de facto standard measurement device to define a fuel's anti-knock quality by means of RON and MON, measured in accordance with the respective ASTM D2699 and D2700 specifications. Knock ratings determined with this protocol provide a ranking relative to binary blends of iso-octane and nheptane, or primary reference fuels (PRFs). In addition, it has been proposed that chemical kinetic models be correlated against octane number in order to validate their accuracy in predicting auto-ignition which would ultimately lead to knock [19] [20]. However, a number of open questions exist concerning the measurements, and how they relate to fundamental processes such as auto-ignition, the development of gas dynamic waves, and so on. 
Swarts et al. conducted a detailed investigation into the role of autoignition during the RON and MON rating methods in an instrumented CFR engine [21]. Their investigations identified a sharp inflection in the cylinder pressure before the onset of high frequency pressure oscillations typically associated with knock. It was hypothesized at the time that this so-called "knock point" could signify auto-ignition of some portion of the end gas, though the ensuing rates of heat release were uncharacteristically slow relative to homogeneous, or volumetric auto-ignition. On the other hand, the pressure rise rate (PRR) following the knock point (KP) appeared to correlate with the reading of the knockmeter for the range of PRF blends tested. Conversely, Arrigoni et al. had previously defined the sharp inflection point in the cylinder pressure trace to be the onset of knock in the CFR engine, while the amplitude of the subsequent high frequency pressure oscillation was found to be smaller than those measured in production spark ignition engines [22]. The high frequency oscillations are typically suggestive of the intensity of knock, and these have been characterized by the magnitude of the maximum oscillation.

The original "bouncing pin” knock indicator was conceived by Midgley and Dickenson for use in the CFR engine [4]. It consisted of a piston and spring pressure element exposed to the cylinder gas pressure on top of which a free pin was placed loosely in a guide with the lower end being in contact with the piston surface. Under knocking combustion the pin was thrown off the piston surface and closed a contact-point to an electrolytic cell, acting as an integrator to capture the knock occurrence over a given time period. Swarts et al. conducted a detailed investigation of the "bouncing pin", the function of which is replicated with the modern D-1 detonation pickup and 501-C detonation meter [23]. The D-1 pickup is a magnetostrictive pressure transducer which generates a voltage proportional to the rate of change of pressure inside the cylinder, while the 501-C detonation meter conditions the signal before passing the voltage to the knockmeter read-out. Swarts et. al. highlighted the use of a low-pass input filter in the circuit diagram of the detonation meter, which indicated that the knockmeter was tuned for frequencies lower than $6.5 \mathrm{kHz}$, making it oblivious to the high frequency pressure oscillations generally associated with knock intensity (KI) [23] Furthermore, a variety of PRF, toluene standardization fuel (TSF), and full boiling range fuels with varying octane numbers were tested in their work, and it was revealed that the PRRs following KP did not agree with the knockmeter reading for the fuels tested. With the aid of a numerical model of the "bouncing pin", it was argued that the knockmeter reacts to the pressure development immediately following the knock point, rather than the average pressure rise rate. Moreover, it was later concluded that the characteristic PRR of a particular knockmeter reading was not consistent, but varied as a function of compression ratio. The METER setting on the 501-C detonation meter serves to scale the signal before displaying on the knockmeter read-out [24]. Finally, it was conjectured that the measured pressure development, under "standard knock intensity", was a result of non-instantaneous cascading auto-ignition, or sequential auto-ignition, as originally described by Zeldovich [25].

While the fundamentals of auto-ignition in the end gas have been investigated for some time [26, 27], and can be predicted fairly consistently with a relatively simple, semi-empirical knock integral model, the knock intensity following the development of autoignition is not as well understood, and has gained much attention in recent times [28, 29, 30,31]. This is in part due to the mainstream application of downsizing and turbocharging in SI engines for efficiency improvements, where this has led to the occurrence of very extreme knock events, termed “super-,” or “mega-knock” [2].
Challenges associated with the accurate measurement and prediction of knock intensity were recently highlighted [28, 30, 31, 32].

The objectives of this study were multi-fold, and included gaining a more thorough understanding of knock measurements in the CFR engine, and in particular, how different approaches to characterize knock can be compared. Parameters that drive various knock processes and associated metrics can then be better quantified. Moreover, opportunities to exploit the CFR engine platform towards the robust characterization of fuels beyond RON and MON conditions could be developed, along with correlations of CFR measurements to KI, and KLSA challenges in modern engines. Towards this, a detailed combustion analysis of the CFR engine was undertaken, first at operation under "standard knock" conditions, according to the ASTM method. Subsequently, mild intake air boosting and temperature elevation beyond the standard ASTM test method were implemented in an effort to increase the operating range and therefore achieve in-cylinder conditions that are more diverse, and representative of modern SI engines. Compression ratio sweeps were conducted over the matrix of intake conditions, covering a range of knock. With the availability of high speed combustion data, comparisons between quantities such as knock point (KP), knock onset (KO), knock intensity (KI), and the standard ASTM knockmeter reading (KU) could be conducted. $\mathrm{KO}$ is defined as the onset of high-frequency pressure oscillations commonly associated with knock. Throughout this manuscript knock intensity will refer to the high-frequency pressure oscillations, while the knockmeter reading will be referred to as such.

The experimental configuration is described next along with details concerning the measurement protocol, and data analysis / postprocessing techniques. The measured results are then presented, with discussions of trends observed in various knock metrics. Finally, the findings of this work are summarized.

\section{Experimental configuration}

\section{CFR F1/F2 engine set up}

Engine experiments were conducted using a Waukesha CFR F1/F2 octane rating engine. The engine is maintained in nearly original condition in order to allow test runs to be conducted according to the research, and motor octane number procedures outlined in the ASTM protocol summarized in Table 1. Further details covering the standard engine geometry and controls can be found in the appendix.

Table 1. Engine operating conditions of RON and MON tests [33, 34]

\begin{tabular}{lcc}
\hline Test & RON & MON \\
\hline ASTM method & D2699 & D2700 \\
\hline Engine speed $(\mathbf{r p m})$ & 600 & 900 \\
\hline Intake air temperature $\left({ }^{\circ} \mathbf{C}\right)$ & $\begin{array}{c}\text { Based on } \\
\text { barometric } \\
\text { pressure }\end{array}$ & 38 \\
\hline Mixture temperature $\left({ }^{\circ} \mathbf{C}\right)$ & Not controlled & 149 \\
\hline Spark timing $\left({ }^{\circ} \mathbf{b T D C}\right)$ & 13 & $\begin{array}{c}\text { Based on } \\
\text { compression } \\
\text { ratio }\end{array}$ \\
\hline Coolant temperature $\left({ }^{\circ} \mathbf{C}\right)$ & 100 & 100 \\
\hline
\end{tabular}


In order to acquire detailed and reliable measurements of engine combustion characteristics and operating conditions, several instrumentation upgrades common for modern engine combustion research have been added to the CFR engine at Argonne National Laboratory. Important to note is that all of these measurement upgrades have taken place without affecting the geometry or performance of the CFR engine during standard octane testing. A list of the upgraded measurement and instrumentation devices is provided in Table 2.

For this investigation the engine was operated in the RON set-up, apart from the use of the heated intake manifold that is employed for the MON test procedure. Furthermore, the engine was coupled to an external charge boosting system, feeding compressed air to the standard carburetors. The standard jet size was maintained and the boosting system was designed to maintain the standard CFR engine air-fuel ratio control by means of adjusting the carburetor bowl height. Once a bowl height and fueling rate was selected at a given boost pressure, the air-fuel ratio remained constant throughout the compression ratio sweep.

In addition to the ASTM knockmeter, which is a relative measurement based on the knocking characteristics of PRFs within one octane number of the test fuel, it is possible to measure absolute characteristics of the knocking combustion using cylinder pressure indicating equipment. While the accuracy limitations of spark plug pressure transducers are well established, the AVL GU13Z-24 nevertheless allows for general analyses of the cylinder pressure and combustion characteristics while simultaneously measuring the ASTM standard knockmeter reading. For part of this investigation, in-cylinder pressure data were recorded at a fixed sampling rate of $200 \mathrm{kHz}$ using an AVL 620 IndiSet data acquisition system. A more accurate and robust, flush-mounted piezoelectric Kistler 6045AU20 pressure transducer was also used for this study, though its installation necessitated the removal of the ASTM knockmeter, as will be outlined in the experimental procedure section.

Table 2. Modern engine combustion research measurements and instrumentation upgrades to the Argonne CFR F1/F2 engine

\begin{tabular}{|c|c|}
\hline \multicolumn{2}{|c|}{ Crankshaft angle-based measurements } \\
\hline Crank-angle based DAQ & AVL IndiCom \& crankshaft encoder \\
\hline Spark timing & Coil wire current clamp \\
\hline Intake pressure & $\begin{array}{l}\text { Kulite high-speed } 2.0 \text { bara pressure } \\
\text { transducer }\end{array}$ \\
\hline Exhaust pressure & $\begin{array}{l}\text { Kulite water-cooled high-speed } 3.5 \\
\text { bara pressure transducer }\end{array}$ \\
\hline Cylinder pressure & $\begin{array}{l}\text { AVL GU13Z-24 Spark plug pressure } \\
\text { transducer }\end{array}$ \\
\hline \multicolumn{2}{|c|}{ High Frequency Data Acquisition } \\
\hline DAQ & AVL 620 IndiSet \\
\hline Cylinder Pressure & Kistler 6045AU20 \\
\hline \multicolumn{2}{|c|}{ Time-based measurements } \\
\hline Time-based DAQ & LabVIEW \\
\hline Intake pressure & Setra 3550 pressure transducer \\
\hline Exhaust pressure & Setra 3550 pressure transducer \\
\hline $\begin{array}{l}\text { Intake, mixture, exhaust, } \\
\text { coolant, and oil temperature }\end{array}$ & K-type thermocouples \\
\hline Fuel rate & Emerson CMF010M \\
\hline Air rate & Alicat mass flow controller \\
\hline Lambda & Bosch wide-band lambda sensor \\
\hline
\end{tabular}

Page 3 of 17

\section{Experimental procedure}

All tests for this investigation were conducted using PRF90, which contained $90 \%$ iso-octane and $10 \%$ n-heptane by volume. The geometric compression ratio of the engine was verified before this study using a hydraulic calibration method where the combustion chamber was filled with SAE30 oil, and the mass of oil recorded at varying clearance heights.

\section{Knockmeter Calibration}

At the start of the test campaign, the knockmeter was calibrated for PRF90 according to the protocol outlined in the ASTM method. The procedure specifies a compression ratio and intake air temperature for a given atmospheric pressure. On this day the barometric pressure reading of 987 mbar implied an intake air temperature (upstream of the carburetor) set-point of $45.3^{\circ} \mathrm{C}$ and a compression ratio of $6.51: 1$ needing to provide a standard knock reading of 50 knock units (KU) on the knockmeter. The knockmeter was calibrated as such, in accordance with the ASTM method, which includes checking the calibration with PRF89 and PRF91 as bracket fuels. The PRF89 fuel needed to result in a knockmeter reading of between 12 and $15 \mathrm{KU}$ above standard knock of 50, while the PRF91 would under-read by the same. Finally, a fit-for-purpose test was conducted using the appropriate toluene standardization fuel (TSF89.3) to ensure that the knockmeter was reading to within $0.3 \mathrm{KU}$ of the 89.3 octane as defined by the ASTM procedure. The engine was found to be fit-foruse without the need for intake air tuning.

The intake air boost system was installed and the supply air pressure was set to 987 mbar. The knockmeter reading was once again checked with PRF90, the bracket PRFs and the TSF89.3 fuel and found to only need a minor METER adjustment. The standard conditions for 987 mbar atmospheric pressure became the "atmospheric reference" condition for all tests going forward.

\section{Test Protocol}

The knockmeter was checked and the data logged at the beginning of each day of testing after the engine was allowed to warm up for at least one hour under "standard knocking" conditions. Thereafter, compression ratio sweeps were conducted as outlined in the test protocol in Table 3 at an air-fuel ratio of $\lambda=1$. At each temperature and boost pressure the compression ratio was swept from 5:1, to "standard knock" of $50 \mathrm{KU}$ and on to a knockmeter reading greater than 65, with the maximum amplitude of the pressure oscillations (MAPO) exceeding 1 bar. MAPO is defined as the maximum amplitude of the pressure oscillations of the bandpass filtered $(6-20$ khZ) cylinder pressure, as shown in Equation 1.

$$
M A P O=\operatorname{maximum}\left\{P_{\text {filtered }}\right\}
$$

The 'real-time' MAPO reading was calculated by means of a rolling average over 100 combustion cycles, using the crank-angle based measurement system. Six compression ratios were recorded at each intake temperature and boost condition resulting in 72 test points.

Table 3 Engine test protocol

\begin{tabular}{lll}
\hline Compression Ratio & Intake Mixture & Intake Boost \\
\hline
\end{tabular}




\begin{tabular}{lcc}
\hline Criteria & Temperature & Pressure \\
\hline $\mathrm{CR} \approx 5: 1-7: 1, \mathrm{KU} 50, \mathrm{KU}>$ & $33,90,150{ }^{\circ} \mathrm{C}$ & $\begin{array}{c}1.0,1.1,1.2,1.3 \\
\text { bar abs. }\end{array}$ \\
65 or MAPO $>1$ bar & & \begin{tabular}{l} 
M \\
\hline
\end{tabular}
\end{tabular}

Time-based data, including the intake pressure, etc., were recorded at a sampling rate of $1 \mathrm{~Hz}$ for a duration of 1 minute while 300 combustion cycles were logged for the crank-angle based measurements. Two recordings were taken at each data point.

Once the compression ratios covering the operating sweep had been determined, the knockmeter was replaced with a Kistler 6045AU20 transducer and the tests were repeated while recording 300 combustion cycles at a sampling rate of $200 \mathrm{kHz}$. The 6045AU20 transducer is an uncooled device designed with a reinforced diaphragm to minimize the effects of thermal shock and steep pressure rise rates on the measurements. Before use, the transducer and the AVL IndiSet measurement chain were calibrated using a Budenberg dead-weight tester over a measurement range up to 100 bar.

\section{Data Analysis}

\section{Crankshaft-based measurements}

Since the $200 \mathrm{kHz}$ data was recorded time based rather than crankshaft resolved and prevented the use of the knockmeter, the AVL GU13Z-24 spark plug transducer was used for real-time combustion data analysis in order to provide guidance on knock intensity achieved at a given compression ratio and knockmeter reading. Furthermore, crank-resolved data from the spark plug transducer is necessary to appropriately perform heat release analyses. It was therefore decided for comparison purposes to conduct measurements at standard RON operating conditions for PRF90 and record data at an interval of 0.1 crank angle degrees (CAD) with both the AVL and Kistler transducers. The aim was to characterize constraints of the AVL spark plug transducer in terms of mounting location within the combustion chamber and thermal shock influences.

Figure 1 presents representative pressure traces for the AVL GU13Z24 in panel (a), with analogous traces recorded using the Kistler 6045AU20 shown in panel (b). The apparent heat release rates (aHRRs) for the combustion cycles are also illustrated. As described and outlined by Swarts and co-workers [21], KP was detected using a 4 point moving average on the pressure trace, and is depicted for all 300 cycles using black circular marks. For PRF90, a pressure rise rate of 3.11bar/CAD was found by Swarts et al. [21] to correlate with KP; in the current dataset this was matched with the Kistler transducer. However, the threshold had to be reduced to $2.5 \mathrm{bar} / \mathrm{CAD}$ for the AVL transducer, since the gradient after knock point was found to be more shallow. This is also evident in the aHRR curves presented here. It is worth noting that the AVL QC32C-X pressure transducer used in the engine by Swarts et al. was flush mounted, and located adjacent to the knockmeter, similar to the location of the Kistler transducer used in this study [21, 23]. So, it is unclear how much the location of the pressure transducer may have had a role in detecting $\mathrm{KP}$ versus the type of transducer.

The onset of knock (KO) for all cycles is shown by the vertical grey hash marks, where these were determined by filtering the pressure traces with a 6 to $20 \mathrm{kHz}$ bandpass filter to calculate MAPO according to Equation 1 and finding the crank angle position where it

Page 4 of 17 first exceeded 0.4 bar. As noted by Kalghatgi et al. there is no standard method to define knock onset [29]. The standard cycle-tocycle deviations for KP and KO are shown using red error bars. There appears to be substantial variability in both the pressure records and associated heat release rates, though the KP pressures are fairly consistent. There is significant scatter in the KO pressures.

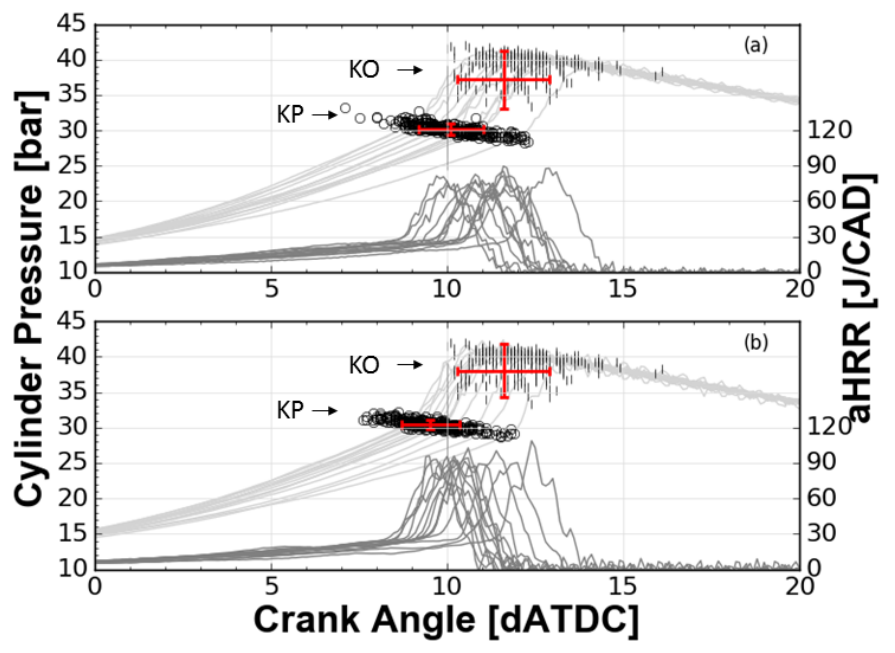

Figure 1 - Representative pressures and heat release rates presented as functions of crank angle, highlighting average and spread of KP and KO measured with (a) AVL GU13Z-24, and (b) Kistler 6045AU20. The engine was operated at "standard knock" of $50 \mathrm{KU}$ with a "peak knocking" $\lambda$ of 0.89, 6.5:1 compression ratio at an intake air temperature of $45.3^{\circ} \mathrm{C}$ at 987 mbar intake pressure.

Figure 2 next illustrates the calculated aHRRs using the two transducers, plotted as functions of the apparent heat release (aHR). The aHRRs were calculated according to Heywood [35], neglecting crevice flow and wall heat loss approximations, but roughly accounting for these effects by subtracting the calculated heat release from analogous skip-fired cycles [17]. The temperature at intake valve closing (IVC) was estimated using an in-house three pressure analysis GT-Power model of the CFR engine [36]. The KP inflection can be seen to occur at roughly $60 \%$ of total aHR which is similar to the conditions observed in previous work [21]. Some cycle-to-cycle variability can be seen in Fig. 2, and this slightly affects the calculated MFB at KP. Also evident is that the peak aHRR during the auto-ignition event is larger for the Kistler 6045AU20 than the AVL GUI3Z-24, where these are identified in the figure. Overall, it appears that the measurements and derived parameters using the AVL GU13Z-24 and Kistler 6045AU20 are fairly consistent. 


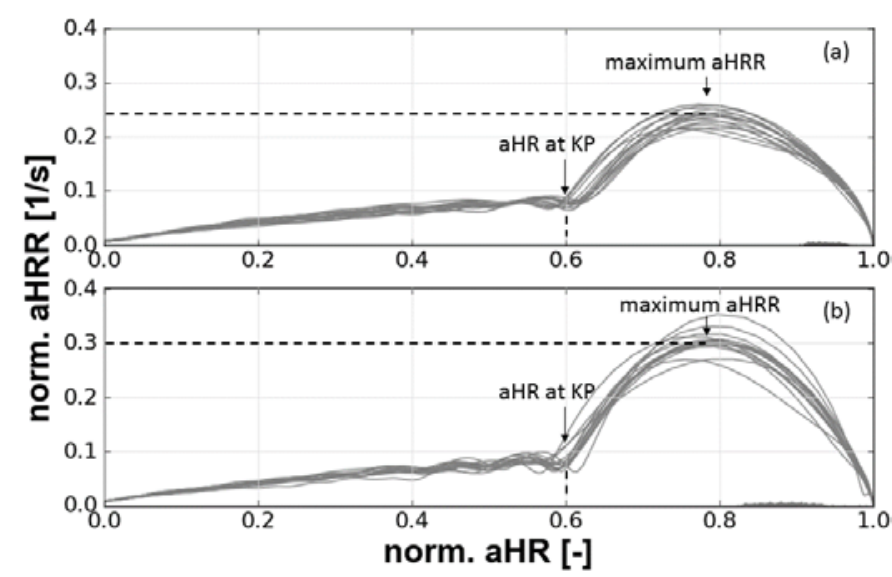

Figure 2 - Representative apparent heat release rates presented as a function of accumulated heat release, normalized against the total accumulated heat released. Panel (a) is based on the AVL GU13Z24, and panel (b) is based on the Kistler 6045AU20. The engine was operated at "standard knock" of $50 \mathrm{KU}$ with a "peak knocking" $\lambda$ of 0.89, 6.5:1 compression ratio at an intake air temperature of $45.3^{\circ} \mathrm{C}$ at 987 mbar intake pressure.

\section{High frequency data analysis}

A post-processing script was written in Python in order to extract pertinent knock metrics from the high-speed data. The procedure followed is shown in Figure 3 and described next. Timing of KP was determined by first filtering the $200 \mathrm{kHz} 6045 \mathrm{AU} 20$ data with a 3 $\mathrm{kHz}$ low-pass filter, where the raw and filtered traces are shown in panel (a). The peak pressure of the filtered trace was noted in each case, as highlighted by the green dot. The second derivative of the cylinder pressure (panel (b)) was used to locate the peak value indicative of the inflection, or KP. The gradient of the red dashed line in (a) highlights the PRR after KP [21, 22, 23]. Knock Intensity was calculated using two methods. The conventional MAPO, according to equation 1 , is shown by means of the high-pass filtered signal in panel (c), which is roughly 1.8 bar for this trace. The second magenta dot in (a) and (c) indicates the time when the maximum pressure amplitude is detected. Values of KI were also ascertained according to the method presented by Shahlari and Ghandhi [32]; shown in panel (d). The blue dots in (d) represent peak oscillations detected during the "ring down" period, which are used to fit an exponential decay function according to the temporal absorption mechanism highlighted by Shahlari and Ghandhi [32] and described using Equation 2 [37] .

$$
K=K_{o} e^{-m t}
$$

The parameter $\mathrm{K}_{\mathrm{o}}$ in the function describes the knock intensity which is then employed to determine the pressure at 400 microseconds after knock onset, as shown by the red dot. The delay period was chosen in order to avoid the potential transducer shock period [32]. For this trace it is apparent that K400 is slightly lower than MAPO and that the pressure oscillation detected by MAPO overshoots the exponential decay fit slightly. Values of $\mathrm{KO}$ were also calculated using two methods. Shahlari and Ghandhi [32] used an iterative method whereby $\mathrm{KO}$ was first estimated and then a fit line was drawn through raw data points 40 microseconds before the estimated $\mathrm{KO}$ and projected forward. Once the pressure difference between the raw data and the projected fit line exceeded $10 \mathrm{kPa}$ it was assigned as the new KO, shown as KO_10kPa with the cyan dot here in (d). In the second method, the standard deviation of the high pass filtered Page 5 of 17 pressure before KP was calculated. Once the signal exceeded two standard deviations, this was taken as the indication for $\mathrm{KO}$, shown by the lime green dot. Both methods were found to accurately detect $\mathrm{KO}$, although the standard deviation method was deemed to be more robust since it did not require a hardcoded pressure deviation. KO was found to closely coincide with KP, though it increased slightly with knock intensity.

$$
K O=P_{\text {Highfiltered }}>P_{\text {Highfiltered }} \pm 2 \sigma
$$
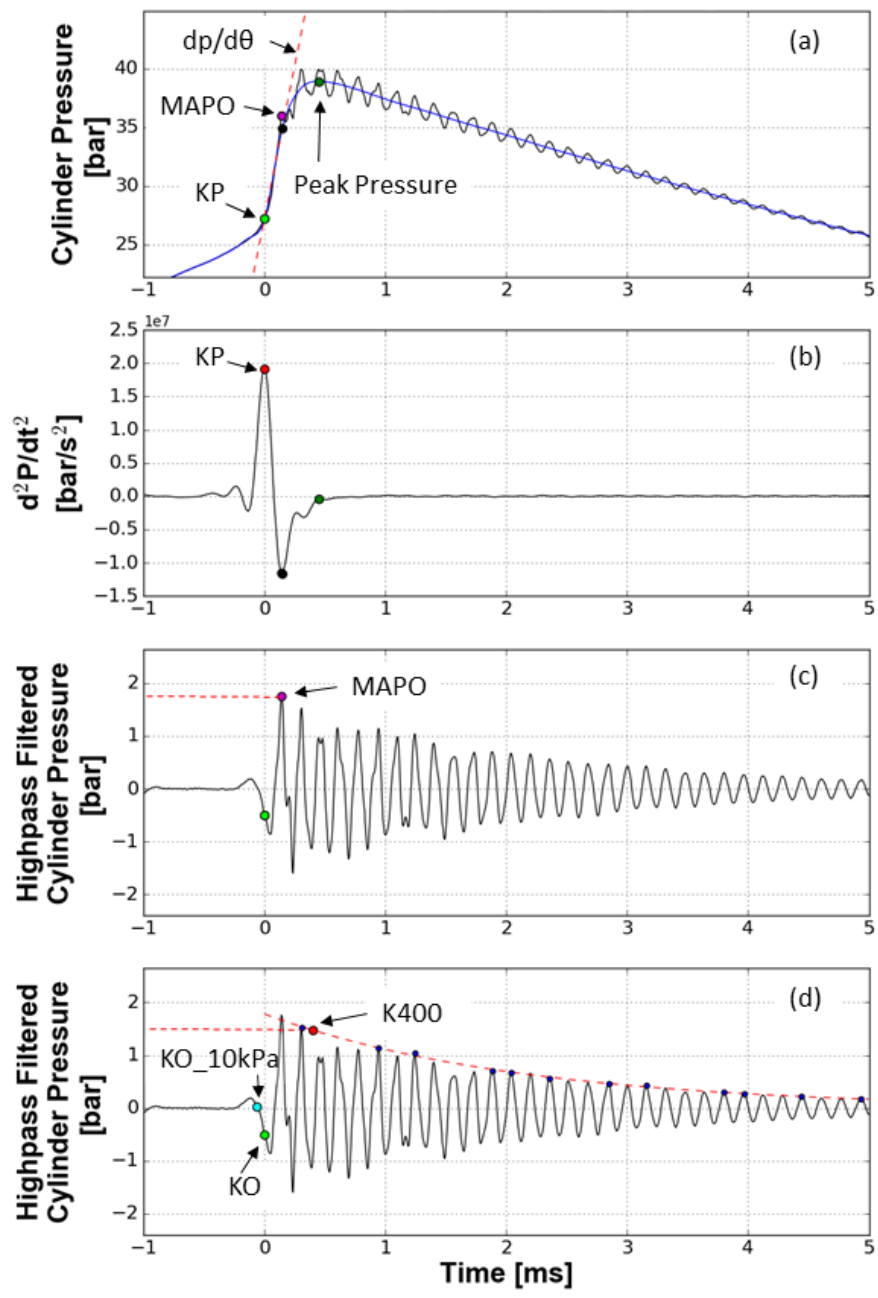

Figure 3 - Definitions of various knock metrics using high speed data. In panel (a) the cylinder pressure signal is shown with the 3 $\mathrm{kHz}$ low-pass filter signal overlaid in blue. Panel (b) depicts the second derivative of the pressure signal used to detect the knock point (KP) and end of the pressure rise rate after KP. Spurious behavior is evident before and after KP as resultant artifacts of the filtering algorithm. Panel (c) depicts the $3-40 \mathrm{kHz}$ band-pass filtered signal used to detect the peak pressure oscillation (MAPO). Panel (d) shows the same band-pass signal used to define K400, as described by Shahlari and Ghandhi [31].

Power spectral density (PSD) analyses were conducted for all operating conditions to calculate the average energy in the frequency domain. This was done over a period of $5 \mathrm{~ms}$ ( 18 CAD) after the onset of knock. The average PSD for 300 cycles is shown in Figure 4 
at the standard ASTM RON conditions. The most dominant frequency detected was $\sim 6 \mathrm{kHz}$; the integrated energy is depicted as the red shaded area under the curve. Other significant frequencies were $\sim 10$ and $\sim 14 \mathrm{kHz}$; the integrated energies for these resonant waves are highlighted in blue and green, respectively. Another peak was detected near $80 \mathrm{kHz}$, but this is the natural frequency of the Kistler 6045AU20 transducer. The total knock spectral energy (KSE) was calculated for each combustion cycle by summing the areas of the three dominant frequencies ( $f$ ), as shown in Equation 4.

$$
K S E=\sum \int_{f 1}^{f 2} P S D f d f
$$

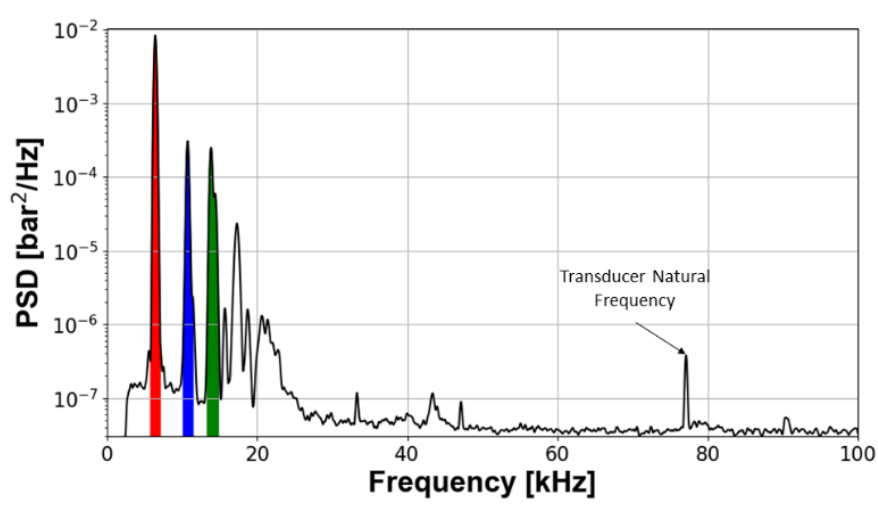

Figure 4 - Power Spectral Density (PSD) analysis of the frequency spectrum for a representative cycle for the Kistler 6045AU20, illustrating the integrated spectral energy at the three dominant frequencies of 6, 10 and $14 \mathrm{kHz}$. The natural frequency of the transducer is also identified.

For each cycle the cylinder pressure at KP, the PRR after KP $(\mathrm{dp} / \mathrm{d} \theta)$, the second derivative of pressure at $\mathrm{KP}\left(\mathrm{d}^{2} \mathrm{p} / \mathrm{d} \theta^{2}\right)$, the pressure at $\mathrm{KO}$, the Knock Intensity (MAPO and K400), the peak filtered pressure, and the KSE were determined. The mean and standard deviation for these are presented and discussed next.

\section{Results and discussion}

Summaries of the measurements across the intake temperature, pressure and compression ratio sweeps, along with the statistics associated with these are illustrated in this section. Black diamond markers are used throughout to depict analogous measurements at the "standard knock" operating point for PRF90, measured according to the ASTM D2699 specification. The knockmeter reading at this condition is $50 \mathrm{KU}$. Error bars are shown at each test point to identify statistical fluctuations.

Figure 5 and Figure 6 show the results for the metrics as functions of compression ratio at all experimental conditions. As noted in the Test Protocol sub-section, the compression ratios were increased upwards from 5.1:1 (where the knockmeter reading was less than 20), through a knockmeter reading of $50 \mathrm{KU}$, to at least $65 \mathrm{KU}$, or the condition where the 'real-time' MAPO exceeded 1 bar. At a few conditions, the knockmeter threshold of $110 \mathrm{KU}$ was exceeded so that increases in compression ratio did not result in a change in the knockmeter reading. This can be seen, for example, for the $33^{\circ} \mathrm{C}$, 1.28 bar intake condition. It should be recognized that since the spark timing was fixed for these sweeps, at $-13^{\circ}$ aTDC, the compressed pressure and temperature at the time of spark increases with compression ratio.

In Figure 5 and Figure 6 it is observed that, in general, most of the monitored quantities increase as the compression ratio increases. Panel (a) first shows the response of the knockmeter. It is interesting to note that the naturally aspirated stoichiometric fuel/air charges tend to require a slightly higher compression ratio than the rich mixtures used at the ASTM conditions to achieve the same reading on the knockmeter. Additionally, it appears that the four different intake pressure conditions are well segregated, where increases in boost pressure cause a reduction in the required compression ratio to achieve the same knockmeter reading. For these sweeps, there does not appear to be a strong dependence on intake temperature however. As noted earlier, due to the monitored knock intensity, the compression ratio sweeps had to be limited in some cases.

Panel (b) shows the pressure at KP where it can be seen that this quantity remains fairly constant across the compression ratio sweep, though there are changes associated with intake temperature and boost pressure. The intake temperature increase reduced the knock point pressure, while intake air boosting conversely, increased the pressure at KP. At the $33^{\circ} \mathrm{C}$ and naturally aspirated operating point, the knock point was not clearly discernable at low compression ratios. These data points are therefore omitted.

Panel (c) next highlights the PRRs after KP which are observed to increase with intake temperature and boost pressure. In general, it appears that higher temperatures led to larger values of $\mathrm{dp} / \mathrm{d} \theta$, while boost pressures seem to do the same, though the trends are not exactly consistent across all of the experimental conditions. For instance, the mixtures at the highest intake temperature do not respond in the same manner to compression ratio, with less increase in $\mathrm{dp} / \mathrm{d} \theta$ for the equivalent change in compression ratio. Furthermore, significant cycle-to-cycle variations are noted at the highest intake temperature, but it unclear why these were observed.

Finally, the peak cylinder pressures are shown in panel (d) where it can be seen that these are somewhat correlated with the level of boost pressure, while intake air heating generally results in lower peak pressures, indicating some influence of changes to the volumetric efficiency during the intake filling process. 

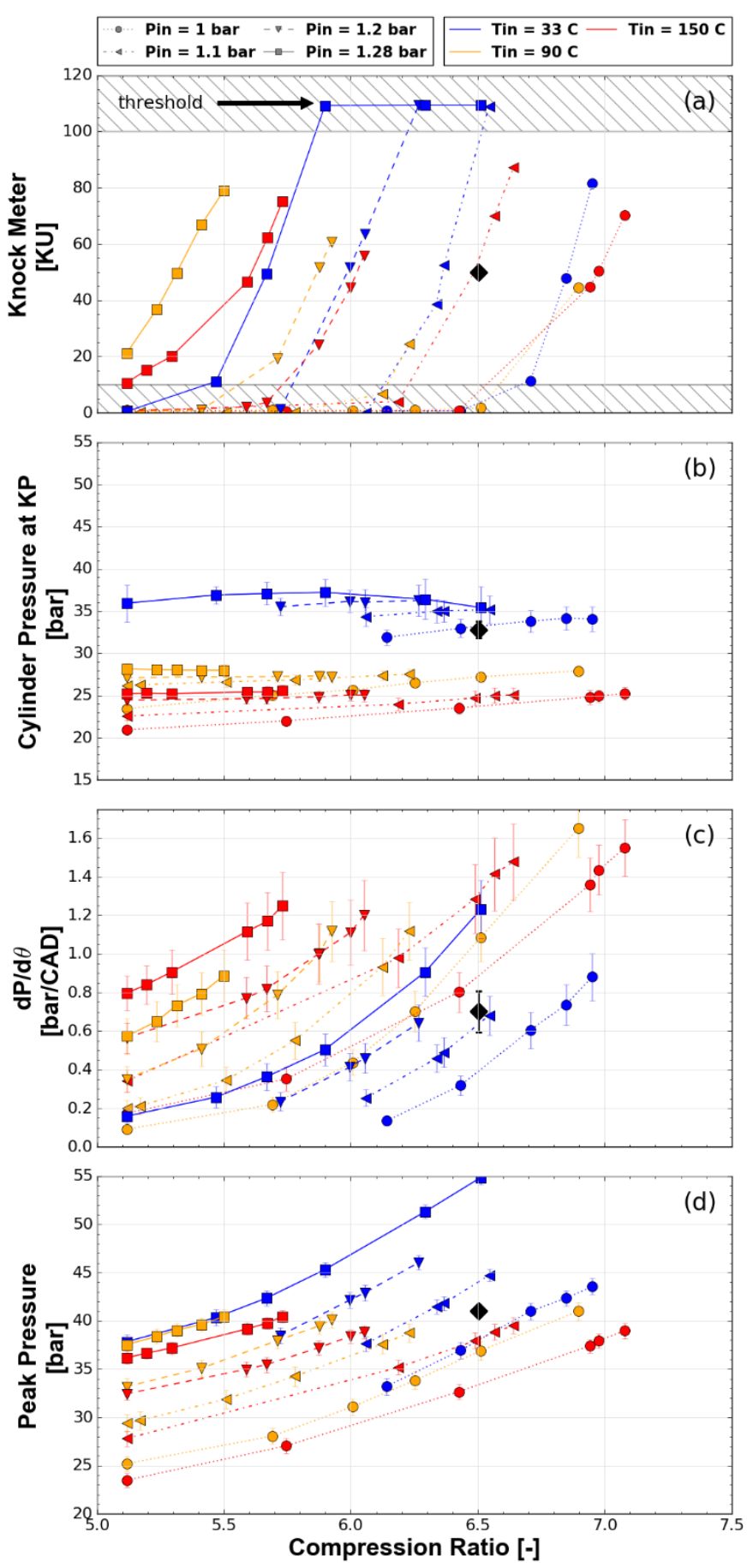

Figure 5 - Response of various knock metrics to compression ratio: (a) Knockmeter reading, (b) pressure at $K P$, (c) pressure rise rate after KP, and (d) peak filtered pressure. 'Standard knock' condition shown as black diamond. Hatched region in panel (a) indicates measurements outside of knockmeter confidence limits, with the knockmeter threshold of $110 \mathrm{KU}$ identified.

Panel (a) in Figure 6 illustrates the trends in pressure at KO, where these are seen to be very similar to those observed for the pressure at KP shown in panel (b) of Figure 5, though the magnitudes are slightly higher, more significantly at higher compression ratios. This suggests that, for these conditions, the knock point coincides closely with knock onset, as previously hypothesized by Swarts et al. [21].
The trends of MAPO and K400, shown in panels (b) and (c), are observed to be very similar, and are quite comparable to those of $\mathrm{dp} / \mathrm{d} \theta$. These features are discussed in greater detail below. At the 33 ${ }^{\circ} \mathrm{C}$ intake temperature, no knock (MAPO) was detected at any boost pressure with the lowest compression ratio of 5.1:1. Moving through the compression ratio sweep, the level of knock intensity increases, where this is exacerbated considerably with the use of charge air boosting. Interestingly, at $90{ }^{\circ} \mathrm{C}$ intake air temperature the knock intensity was at times found to be higher than at $150{ }^{\circ} \mathrm{C}$. This could be related to a lower charge density due to reduced volumetric efficiency, although the knock intensity for the 1.28 bar case was distinctly higher at $150{ }^{\circ} \mathrm{C}$. In addition, for the $90^{\circ} \mathrm{C}$ naturally aspirated case, it was not possible to achieve the standard knock number reading of $50 \mathrm{KU}$ by the condition where the knock intensity reached a mean value of 3 bar, with occasional cycles exceeding 8 bar over-pressure.

The black diamond marker representing the standard ASTM test conditions, shows a knock intensity of approximately 0.6 bar, which, interestingly, is close to the $1 \mathrm{bar} / 1000 \mathrm{rpm}$ limit generally prescribed by automotive original engine manufacturers (OEMs) when calibrating knock control systems. However, at boost pressures of 1.2 and 1.28 bar and temperatures of 90 and $150^{\circ} \mathrm{C}$, a high knock intensity was measured at the lowest compression ratio. Furthermore, it is evident that compression ratio had less influence on $\mathrm{KI}$ at $150{ }^{\circ} \mathrm{C}$ intake air temperature.

Finally, panel (d) shows the KSE measured at each compression ratio, and these are seen to follow the trends observed with knock intensity. This is discussed in more detail below. 

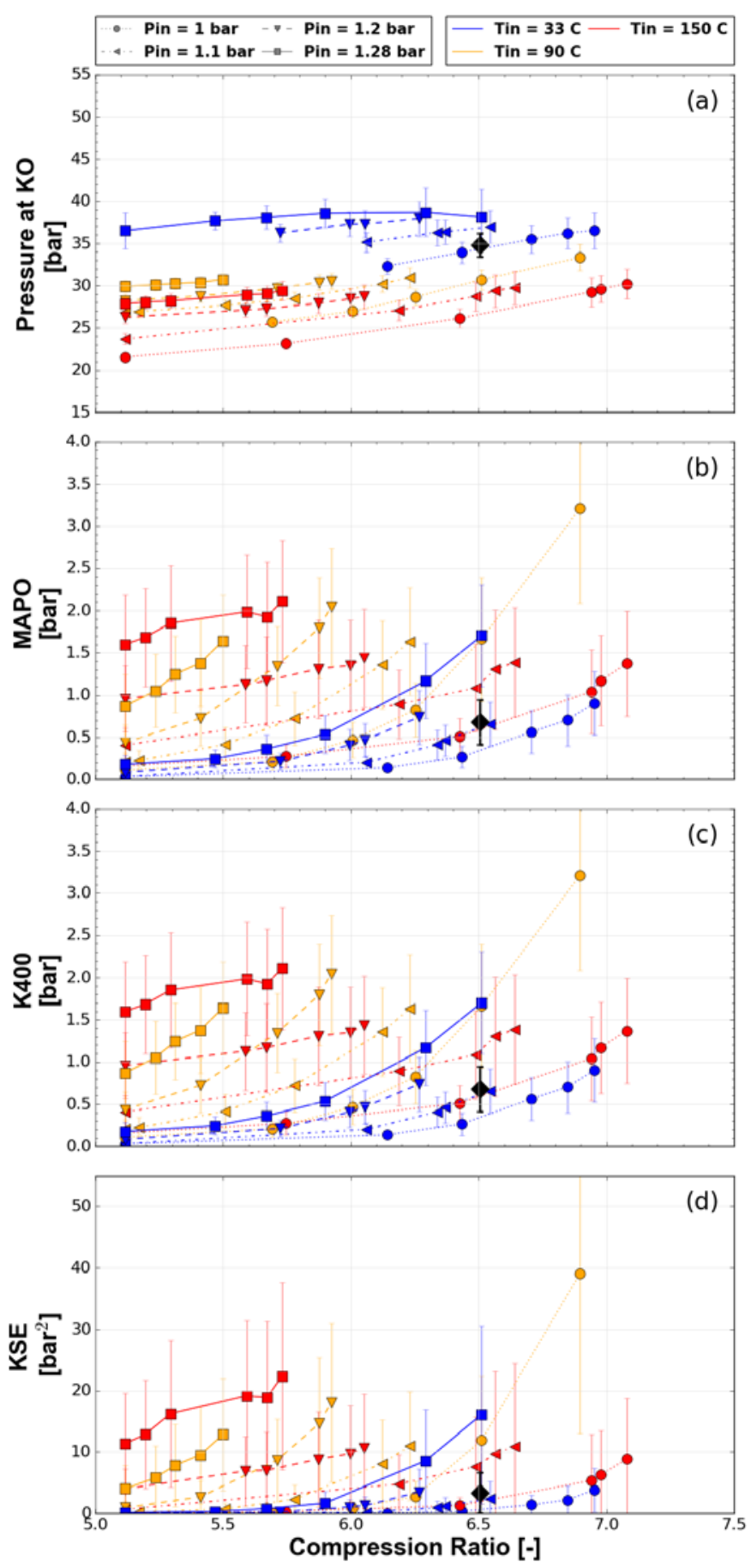

Figure 6 - Response of various knock metrics to compression ratio: (a) pressure at KO, (b) MAPO, (c) K400, and (d) total KSE. 'Standard knock' condition shown as black diamond.

Figure 7 and Figure 8 next organize the results as functions of knockmeter reading covering all experimental conditions. This is done to identify and highlight trends in the knock metrics, and how they relate to the knockmeter reading. Knockmeter readings below 10 and above $100 \mathrm{KU}$ are hatched to indicate that the measurements are outside the confidence limits of the meter. Global trends are provided for each parameter, as shown by grey dashed lines with $\mathrm{R}^{2}$ values identified, and these will be discussed in more detail.
In Figure 7 the pressure at KP is shown in panel (a). While pressure at KP remained fairly constant across the compression ratio sweeps, as shown in Figure 5, the knockmeter reading continued to increase. This suggests that the correlation between the knockmeter reading and $\mathrm{KP}$ is weak.

Panel (b) in Figure 7 illustrates the variation in pressure rise rates at $\mathrm{KP}$ with knockmeter, and contrary to pressure at KP, there is an overall increase in $\mathrm{dp} / \mathrm{d} \theta$ with knockmeter. In general though, greater values are recorded at higher temperatures, while boost pressure leads to lower values of $\mathrm{dp} / \mathrm{d} \theta$ for the corresponding knockmeter readings.

The second derivative of pressure at KP is shown in panel (c), and the trends are fairly consistent with $\mathrm{dp} / \mathrm{d} \theta$. There is not a strong trend with knockmeter reading.

Finally, panel (d) in Figure 7 presents the peak filtered pressure as a function of knockmeter reading. As highlighted by Swarts et al. [23], the knockmeter is equipped with a $6.5 \mathrm{kHz}$ low pass filter, so it is logical to consider the low frequency response of the sensors used in this work. Surprisingly, there appears to be a relatively good correlation between the peak pressure and knockmeter reading, though there is some scatter across the different intake temperatures used in this work, with higher pressures recorded at the lowest temperature. 

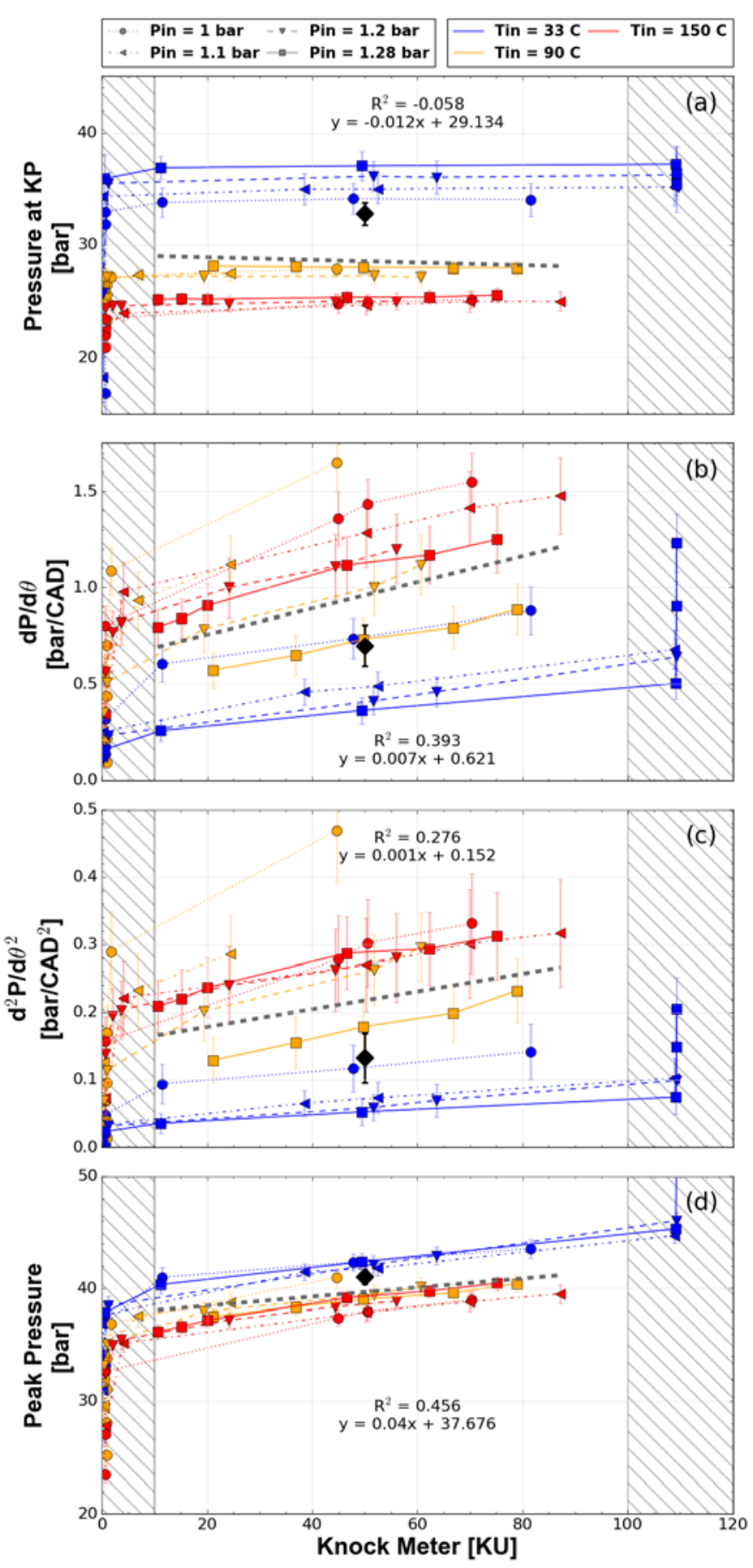

Figure 7 - Trends of various knock metrics with Knockmeter reading: (a) pressure at $K P$, (b) pressure rise rate after $K P$, (c) second derivative of pressure at $K P$, and (d) peak filtered pressure.

'Standard knock' condition shown as black diamond. Hatched regions indicate measurements outside of knockmeter confidence limits. Global trends shown as grey dashed lines with linear fit and $R^{2}$ values identified.

Pressure at KO is shown in panel (a) of Figure 8, where the trends are again, closely aligned with those of pressure at KP as seen in Figure 5 and Figure 6.

Page 9 of 17
Knock intensity is shown in terms of knockmeter reading for MAPO in panel (b) and K400 in panel (c). The trend of lower knock intensity with increasing boost pressure at $33{ }^{\circ} \mathrm{C}$ implies that for a given knockmeter reading the boosted operating conditions have a lower knock intensity. However, in Figure 6 the knock intensity was shown to increase with boost pressure during the compression ratio sweeps, implying that this was not picked up by the knockmeter. The same description applies to the boost pressure trends at $90^{\circ} \mathrm{C}$ intake temperature, while the relation for the $150{ }^{\circ} \mathrm{C}$ intake air temperature is not so clear in terms of knockmeter reading. Finally, as previously discussed, the total KSE trends similarly as MAPO and K400.

As mentioned earlier, it has been suggested in the literature that KP could indicate the timing of auto-ignition in the end gas [21] although it was later concluded that the pressure rise rate after KP was not deemed to be sufficiently high to characterize auto-ignition [23]. It was further suggested that the pressure rise rate immediately following the knock point could affect the knockmeter reading. The current results do not seem to support that hypothesis. However, a detailed model of the original "bouncing pin" mechanism, after which the ASTM Model 501-C detonation meter was designed, suggested that the knockmeter would not be able to detect high pressure oscillations [23] and this agrees with the lack of correlation between the knockmeter reading and MAPO or K400 in this work. The similar trends of pressure at KP and KO further suggest that KP is the onset of knock and that it is not detected by the knockmeter. 

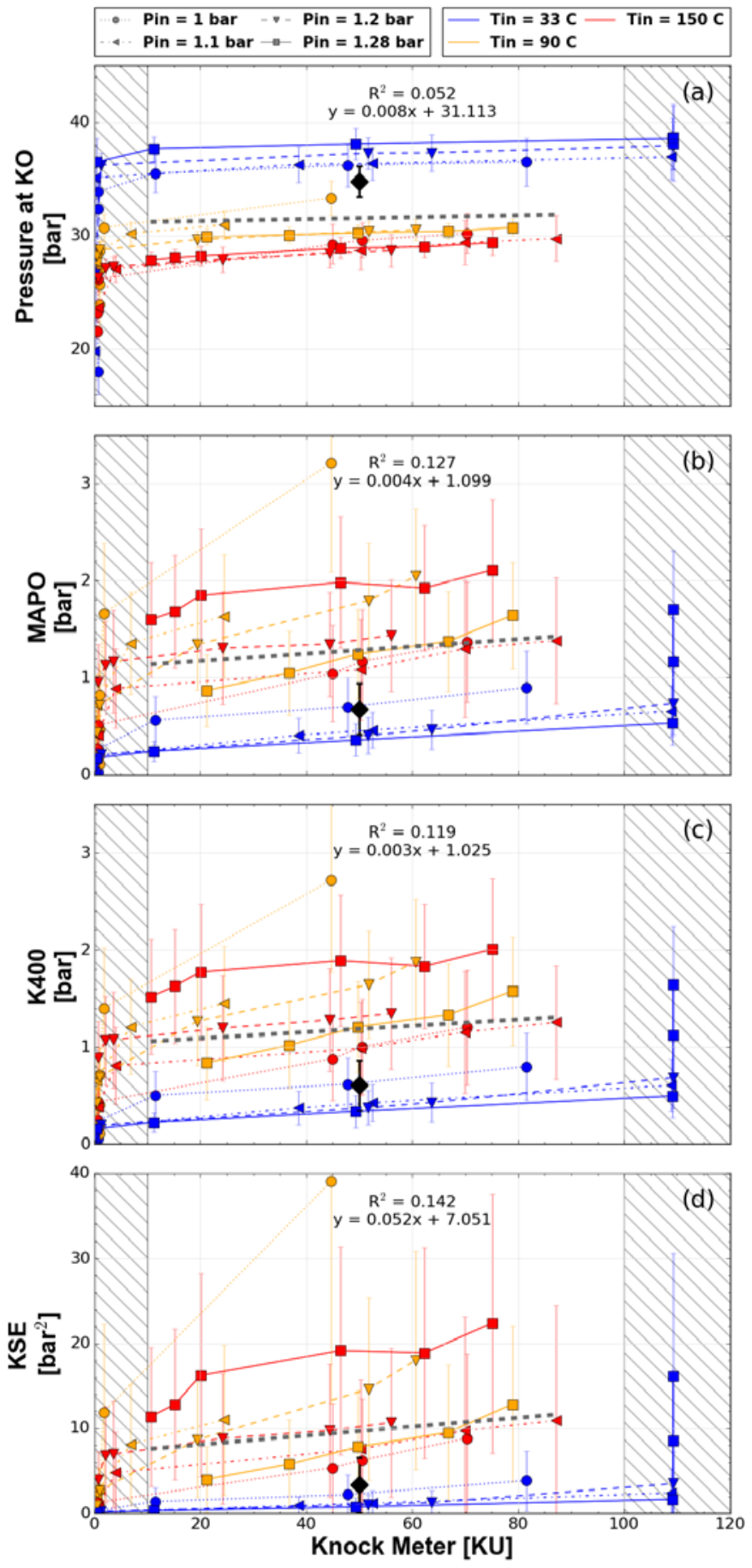

Figure 8 - Trends of various knock metrics with knockmeter reading: (a) pressure at KO, (b) MAPO, (c) K400, and (d) total KSE.

'Standard knock' condition shown as black diamond. Hatched regions indicate measurements outside of knockmeter confidence limit. Global trends shown as grey dashed lines with linear fit and $R^{2}$ values identified.

Three primary frequencies were detected during the PSD analysis for all the test cases, as highlighted in Figure 4. The KSE for the most dominant frequency at $6 \mathrm{kHz}$ is shown in panel (a) in Figure 9. No clear trend was established which implies that, despite being passed through the $6.5 \mathrm{kHz}$ low pass filter to condition the knockmeter signal, the knock spectral energy does not appear to have a strong Page 10 of 17 correlation with the knockmeter reading. The KSE measurements for the $10 \mathrm{kHz}$ and $14 \mathrm{kHz}$ test cases also did not show any correlation as is apparent in panels (b) and (c), respectively. It is important to note the wide range of spectral energies covered in these plots, where more than four orders of magnitude are spanned.
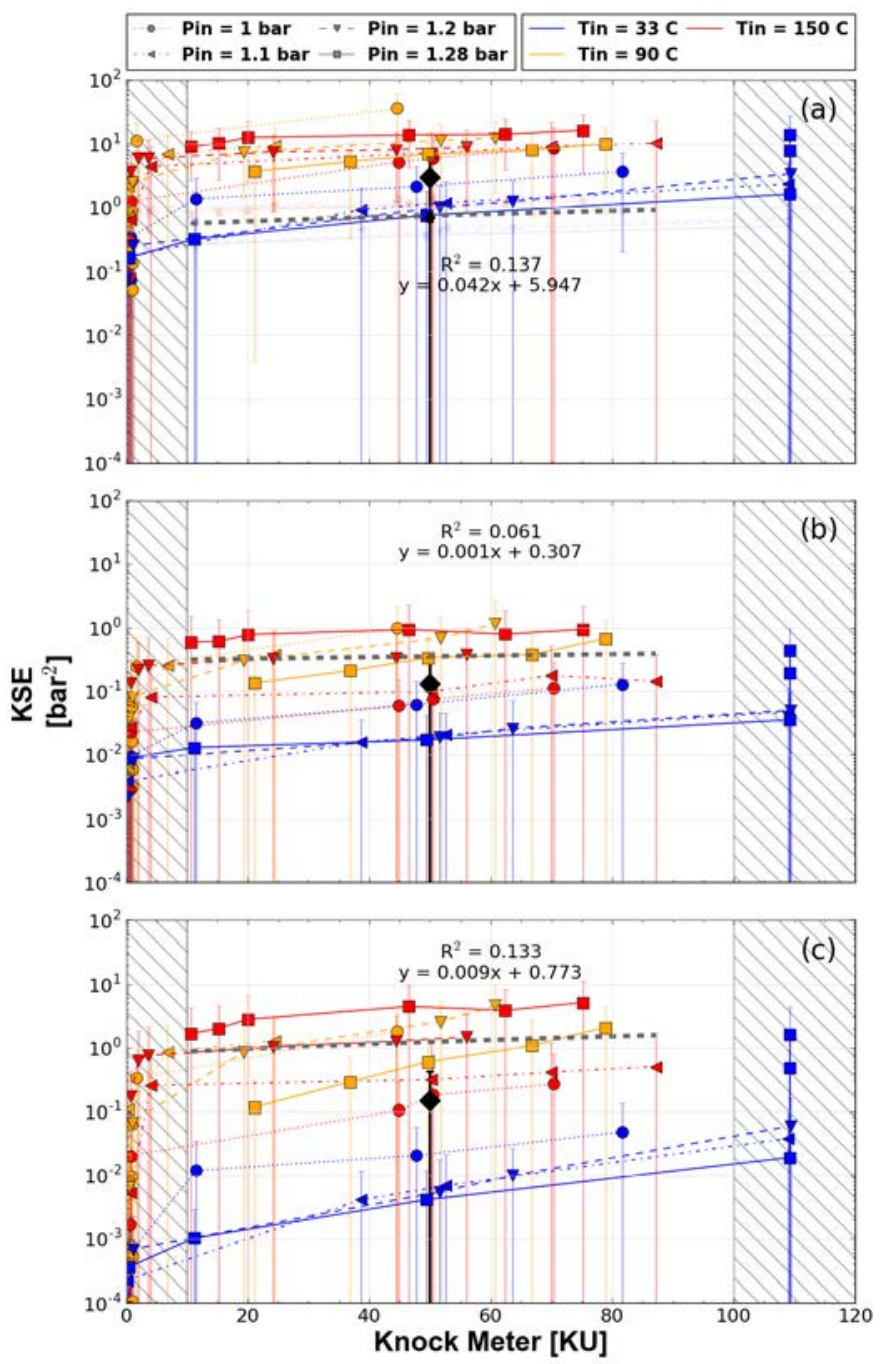

Figure 9 - Trends of frequency-specific/eigen-mode-specific KSE with knockmeter reading: (a) $6 \mathrm{kHz}$, (b) $10 \mathrm{kHz}$ and (c) $14 \mathrm{kHz}$. 'Standard knock' condition shown as black diamond. Hatched regions indicate measurements outside of knockmeter confidence limit. Global trends shown as grey dashed lines with linear fit and $R^{2}$ values identified.

In Figure 10 and Figure 11 the knock metrics are compared against KI as described by MAPO. Panel (a) in Figure 10 depicts the knockmeter reading. It is interesting to note that the shapes of the trends appear to be similar to the correlation with compression ratio shown in panel (a) in Figure 5. Similar to the compression ratio, there appears to be a sudden increase in knock number at a given MAPO threshold. However, the threshold varies with temperature and pressure, and does not follow the same trend seen with compression ratio. The pressure at KP does not have any correlation with MAPO as shown in panel (b). This might be expected since the pressure at KP was shown in Figure 5(b) to be unaffected by changes in compression ratio, even though there were distinct increases in knock intensity. On the other hand, the pressure rise rate after KP does 
appear to correlate fairly well with MAPO; though some deviations are observed across the different intake temperatures. Since the onset of KP and KO have been shown to closely coincide, this implies that the pressure rise rate after $\mathrm{KP}$ or $\mathrm{KO}$ is closely coupled with the generation and development of pressure waves within the combustion chamber, as quantified by knock intensity.

The filtered cylinder pressure is shown in panel (d). In general, as MAPO increases, there is a corresponding increase in peak pressure, however there are significant differences across the three intake temperatures investigated here.
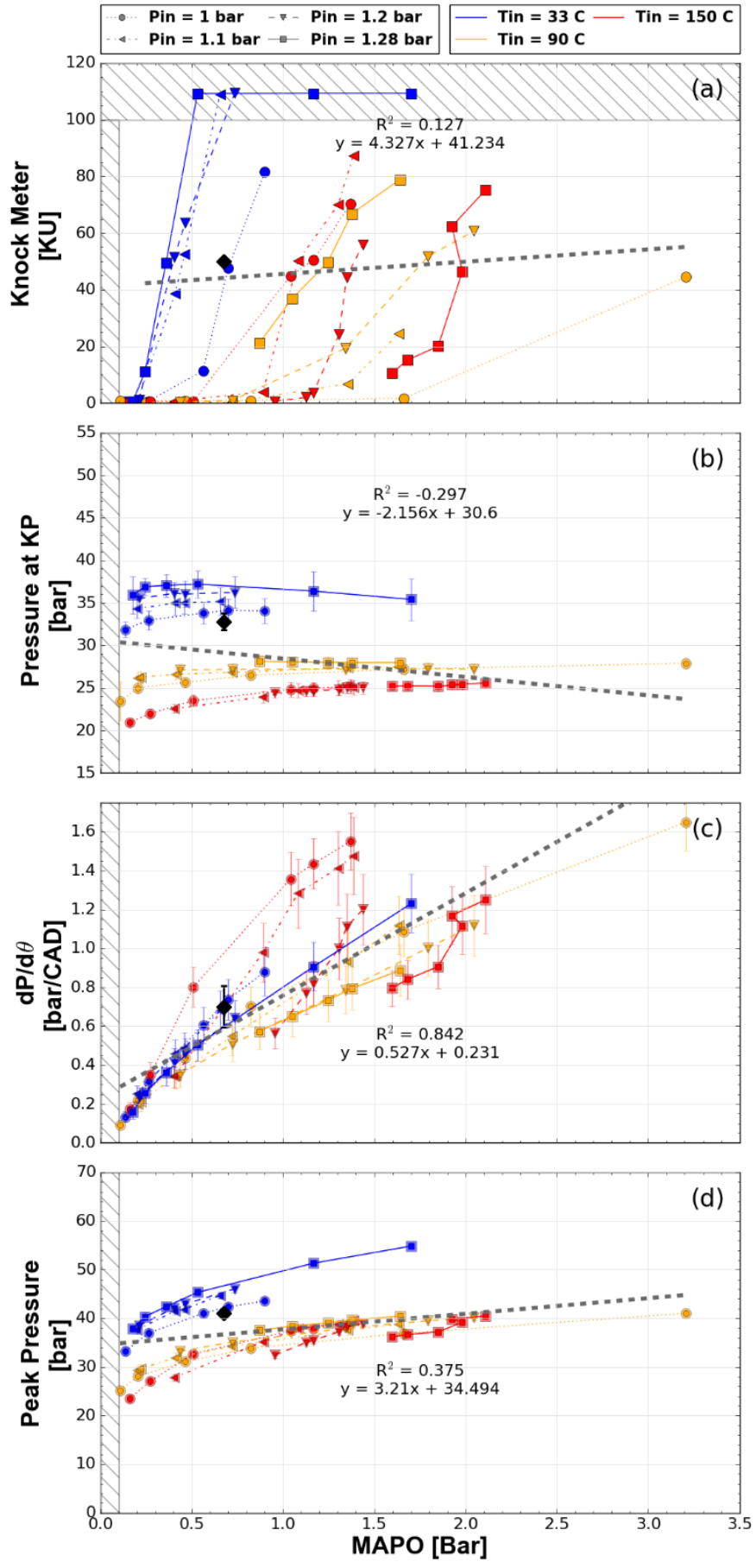

Figure 10 - Trends of various knock metrics with Knock Intensity: (a) Knockmeter reading, (b) pressure at $K P$, (c) pressure rise rate at $K P$ and (d) peak filtered pressure. 'Standard knock' condition shown as black diamond. Hatched regions indicate measurements outside of knockmeter, or MAPO confidence limits. Global trends shown as grey dashed lines with linear fit and $R^{2}$ values identified.

As discussed, a similar trend with pressure at KO and MAPO was found with pressure at KP, and this is observed when comparing panel (a) of Figure 11 and panel (b) of Figure 10. The knock intensity described by K400 was found to agree very well with MAPO for the conditions covered in this work, and this is seen in panel (b) of Figure 11. This finding is different than that observed by Shahlari and 
Ghandhi [32], and could be due to the simple, nearly cylindrical combustion chamber design found in the CFR engine, as well as the lower engine speed conditions used in the current work. The nearly exact correlation between K400 and MAPO provides confidence in the use of MAPO as a metric for knock, since there is good correspondence between the initial peak measured pressure disturbance, and the later stages of knock where the pressure waves resonate.

This feature is further quantified when KSE is compared against MAPO across the conditions studied in this work. A strong correlation is also found between these parameters, as can be seen in panel (c). This implies that the knock intensity, as quantified by MAPO, which is defined at one given point during the cycle, appears to accurately encompass the spectral energy that resonates due to knocking combustion.

A fairly strong correlation was also found with the normalized apparent heat release (aHR) at KP, shown in panel (d). The negative slope indicates that in situations where less of the charge is consumed by the flame, and more energy is released through auto-ignition, greater knock intensity will result.
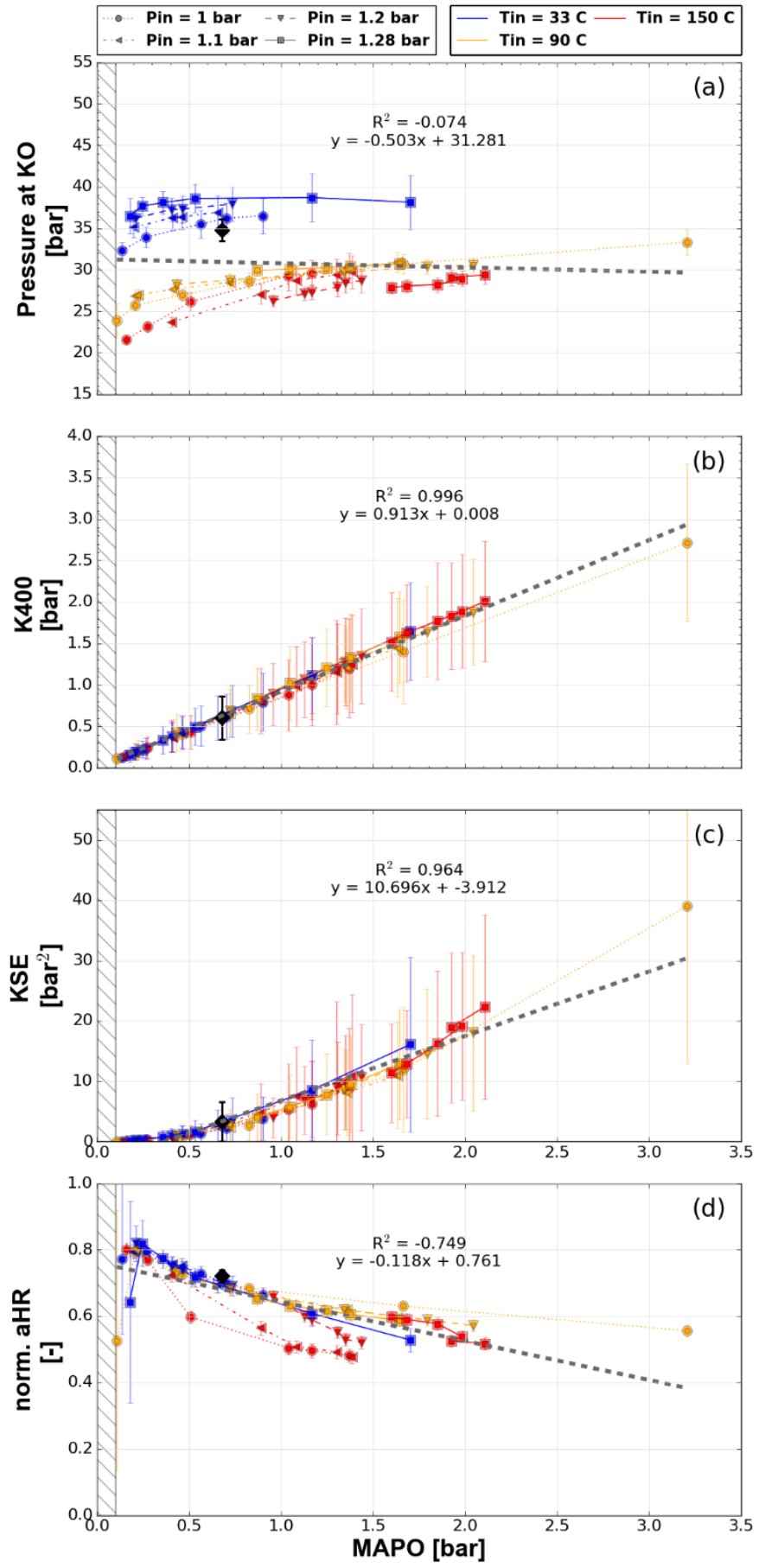

Figure 11 - Trends of various knock metrics with Knock Intensity: (a) pressure at KO, (b) K400, (c) total KSE and (d) normalized aHR. 'Standard knock' condition shown as black diamond. Hatched regions indicate measurements outside of MAPO confidence limit. Global trends shown as grey dashed lines with linear fit and $R^{2}$ values identified.

\section{Discussion}

The global trends in knock metrics presented in Figure 7 to Figure 11 are indicated by dashed lines across the twelve operating conditions. These are summarized in Figure 12 by means of a Pearson productmoment correlation which highlight the respective correlations with 
knockmeter reading and MAPO. Included in Figure 12 are aHR at $\mathrm{KP}$ and peak aHHR at auto-ignition, as identified in Figure 2 and Figure 11. Note that the correlations are ranked from strongest to weakest where positive values indicate that as the parameter increases, there is a corresponding increase in Knockmeter reading, or MAPO, while negative values indicates that there is a corresponding decrease with an increase in the parameter.
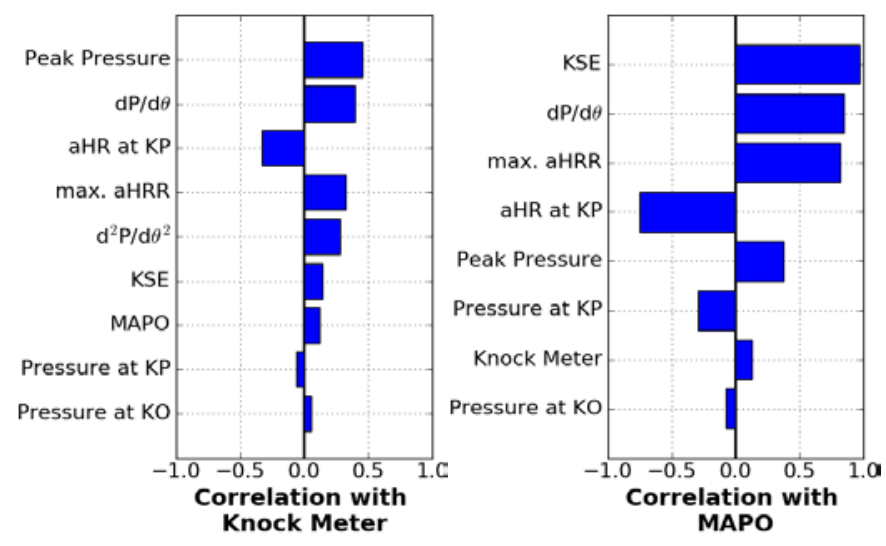

Figure 12 - Pearson product-moment correlation coefficients of various knock metrics presented as functions of Knockmeter and MAPO. Listings are ranked from largest to smallest.

In this work, contrary to earlier publications, it appears that the knockmeter measurement has the highest correlation with peak filtered pressures during a particular test, although the Pearson coefficient of 0.45 (which corresponds to the $\mathrm{R}^{2}$ value listed in Figure 7(d)) is somewhat low. Peak cylinder pressures are dependent on both the timing of end gas auto-ignition (characterized in this work by the pressure at KP), as well as the pressure rise rate during the subsequent auto-ignition process, (characterized here by $\mathrm{dp} / \mathrm{d} \theta$ ). The pressure rise rate during auto-ignition shows a fairly weak correlation with knockmeter reading, while the apparent heat release at the knock point (aHR at KP) is inversely correlated, albeit also with a weak correlation. However, directionally the correlation of pressure rise rate and mass fraction burnt at KP agree with the overall observations since the maximum aHRR after knock point is affected by the remaining mass fraction for auto-ignition, as discussed next. The remaining knock metric parameters are shown to have negligible correlation with the knockmeter reading.

The Pearson correlations for MAPO indicate that there is a near-unity correlation with KSE which suggests that these parameters describe the same phenomena. There are strong correlations between MAPO and pressure rise rate after KP, as previously highlighted in Figure 10 and Figure 11, and peak aHRR at auto-ignition. (It is important to note that aHRRs are computed using the filtered pressure signal, so these parameters are expected to be tightly coupled.) The apparent heat release, or mass fraction consumed, at KP is inversely correlated similar to the correlation with the knockmeter, albeit with a significantly stronger correlation of around 0.75 . The knockmeter reading and peak pressure have weak correlations with MAPO, while weak inverse correlations to pressure at $\mathrm{KP}$ and $\mathrm{KO}$ are found.

The issue of aHRR at auto-ignition is explored in Figure 13 where the aHRR is presented as a function of aHR for a range of conditions where the knockmeter reading was $50 \mathrm{KU}$. As with Figure 2, the data are normalized to the maximum heat released during the cycle in order to ease the interpretation. Five representative consecutive combustion cycles are shown for each test condition.

First, it is interesting to note that the aHRRs during the flame portion of the combustion process are very similar indicating that the burn rate may not be a strong function of the intake conditions. At KP however, the situation changes and it is evident that the aHRRs increase both with intake temperature and boost pressure.

Furthermore, KP can be seen to shift from roughly $70 \% \mathrm{MFB}$ at $33^{\circ} \mathrm{C}$ to around $40 \% \mathrm{MFB}$ at $150{ }^{\circ} \mathrm{C}$. This perspective thus provides additional insight into KP, as compared to the quantification of pressure at KP. For instance, at each temperature, an increase in boost pressure is seen to delay KP, in terms of MFB, as could be inferred from earlier discussions of pressure at KP. This contradicts the general perception that boosted charges are more reactive, and could be due to the different compression ratios used for each of these tests. Since all test cases shown here have a knockmeter reading of $50 \mathrm{KU}$, it can be deduced that, for the test matrix and conditions used in this study, the unburned mass fraction at KP does not directly affect the knockmeter reading. This seems to contradict the conclusions of Swarts et al. [21], though their work utilized fuels covering a range of octane ratings. Figure 13 seems to demonstrate that the peak aHRRs experienced under the current conditions also do not correlate strongly with knockmeter reading.

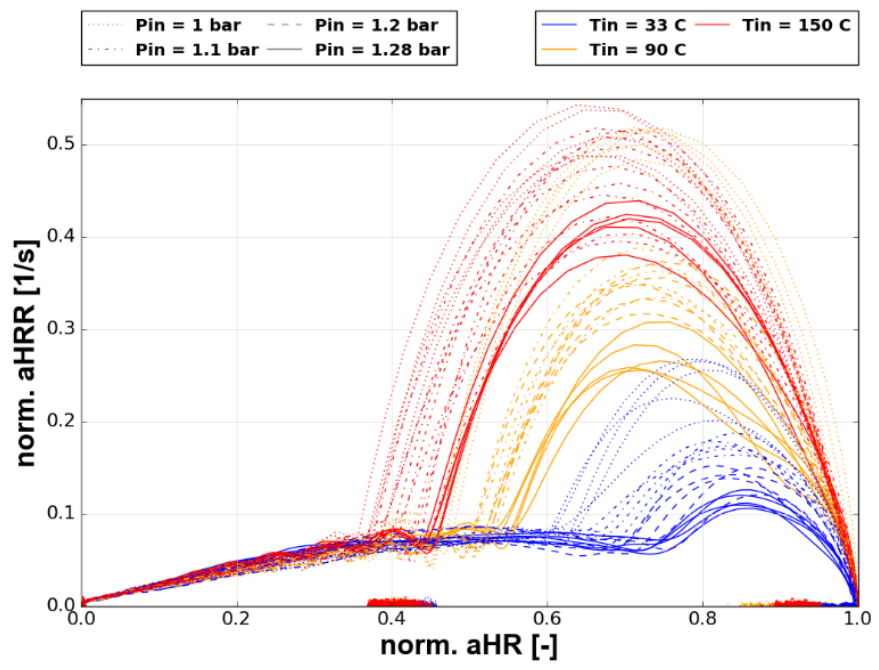

Figure 13 - Normalized apparent heat release rates presented as a function of accumulated heat release for operating conditions where knockmeter reading is $50 \mathrm{KU}$. Five successive combustion cycles are presented for each operating point

End gas auto-ignition is dependent on complex low temperature chemistry that can be very sensitive to temperature, pressure, equivalence ratio and/or dilution. This is complicated by the influence of residual gases such as NOx (which can be quite high under the current test conditions). The local conditions are therefore quite influential on the chemical induction process. Sequential autoignition that may emanate from a reactive center (e.g., hot-spot) is dependent on bulk distributions and gradients across the combustion chamber, as well as the local rates of heat release that occur at autoignition [38]. As such, it seems to be quite possible for two fuels to yield the same knockmeter reading, and thus the same octane rating, where one fuel autoignites early, but has a very slow pressure rise rate, while another autoignites late, but has a more rapid dp/d $\theta$. A previous investigation by Kolodziej and Wallner [39] highlighted this type of behavior and it will be the focus of a detailed follow-up study. 
Such behaviors can be complicated further considering charge densities, heating values of the fuels, and burn rate effects due to fuel composition, vaporization enthalpies, and cylinder temperature and pressure.

Examples of such behavior are shown in Figure 14, where a comparison between representative cylinder pressure traces for three different operating conditions is shown. The blue line depicts the combustion behavior at $33^{\circ} \mathrm{C}$ and the red pressure trace at $150{ }^{\circ} \mathrm{C}$ intake air temperature with a boost pressure of 1.2 bar. The KP occurs significantly earlier at the higher intake temperature and the pressure rise rate after KP is much steeper, subsequently achieving peak pressure earlier, with higher pressure oscillations. As with Figure 13 the knockmeter reading was $50 \mathrm{KU}$ for both cases. For reference, the black line depicts the cylinder pressure measured under standard RON conditions, which by definition had $50 \mathrm{KU}$.

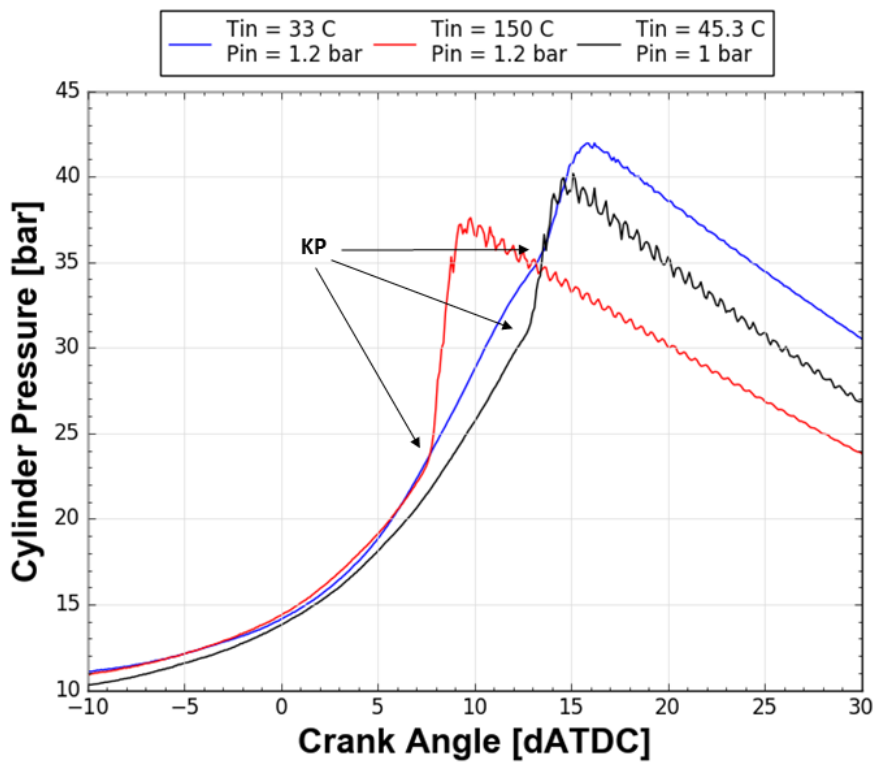

Figure 14 - Representative individual cycles of the low and high inlet temperature conditions at an intake pressure of 1.2 bar and a knockmeter reading of $\sim 50 \mathrm{KU}$. The compression ratio is $\sim 6.0$. The 'standard knock' condition is also shown for reference where Tin = $45.1^{\circ} \mathrm{C}$ and compression ratio is 6.5 .

It is important to note that the $\mathrm{T}_{\text {in }}=33$ and $150{ }^{\circ} \mathrm{C}$ test cases shown here, as well as the pressure and temperature sweeps presented in this work explore different regimes of auto-ignition chemistry and knocking combustion characteristics for a single fuel. From the results it seems apparent that none of the auto-ignition chemistry effects are adequately captured by the knockmeter, and by implication the RON or MON method. It is prudent that these findings be investigated using multi-fuel component blends.

In closing, due to these complex chemicophysical interdependencies highlighted through this work, it seems challenging to properly model the processes that occur within the CFR engine, either in a reducedorder fashion, or via detailed multi-dimensional perspectives to achieve a robust, 'virtual' octane predictor.

\section{Summary}

A study was conducted using the CFR engine to investigate a variety of knock metrics under both a standard ASTM test condition, and compression ratio sweeps that were conducted across a range of intake temperature and boost pressure conditions. The findings can be summarized as follows:

- The KP was detected over the complete operating map, traversing a range of intake pressure and temperature conditions.

- The pressure at which KP occurs was weakly a function of intake pressure while being more influenced by the intake temperature.

- The cylinder pressure at KP was found to be fairly constant throughout the compression ratio sweeps and did not correlate with knock intensity or knockmeter reading.

- $\quad$ The cylinder pressure at KP was found to closely correlate with KO, supporting the initial hypothesis by Swarts et al. [21] that KP indicated the onset of knock in the CFR engine.

- Accordingly, knock intensity also did not correlate with cylinder pressure at $\mathrm{KO}$, implying that it was not directly driven by pressure.

- $\quad$ The pressure rise rate after the KP was found to increase somewhat with intake boost pressure and temperature. No direct correlation could be established with the knockmeter although pressure rise rate did correlate quite well with knock intensity.

- Knock intensity defined by MAPO was found to directly correlate with the alternative method presented by Shahlari and Ghandhi [31]. The pressure transducer shock period appeared to be negligible under the operating conditions investigated in the CFR engine.

- The KSE was found to correlate well with knock intensity (MAPO and K400), implying that the single value in the knock intensity metric captured most of the characteristics of the accumulated energy spectrum.

- $\quad$ No correlation to the knockmeter reading was found between the knock spectral energy of the three dominant frequencies.

- $\quad$ The unburned mass fraction was not found to correlate with the knockmeter reading.

- At a fixed knockmeter reading of $50 \mathrm{KU}$, intake air heating shifted the KP from roughly 70 to $40 \%$ MFB.

\section{References}

[1] J. Warnatz, U. Maas and R. W. Dibble, Combustion: physical and chemical fundamentals, modeling and simulation, experiments, pollutant formation, Berlin: Springer, 2006.

[2] G. Kalghatgi, Fuel/Engine Interactions, Warrendale: SAE International, 2014.

[3] H. Ricardo, "Recent Research Work on the InternalCombustion Engine," Society of Automotive Engineers, no. 220001, 1922. 
[4] T. Midgley and T. A. Boyd, "Methods of Measuring Detonation in Engines," Society of Automotive Engineers, no. 220004, 1922

[5] H. K. Cummings, "Methods of Measuring the Antiknock Value of Fuels," Society of Automotive Engineers, no. 270003, 1927.

[6] G. Edgar, "Comparison of Methods of Measuring Knock Characteristics of Fuels," Society of Automotive Engineers, no. 280008, 1928.

[7] G. Edgar, "Detonation Specifications for Automotive Fuels," Society of Automotive Engineers, no. 270006, 1927.

[8] N. Mac Coull, "Methods of Measuring Detonation," Society of Automotive Engineers, no. 280007, 1928.

[9] E. Bartholomew, "New Knock-Testing Methods needed to match Engine and Fuel Progress," Society of Automotive Engineers, no. 610200, 1961.

[10] G. T. Kalghatgi, "Fuel Anti-Knock Quality - Part2. Vehicle Studies - How Relevant is Motor Octane Number (MON) in Modern Engines?," SAE International, no. 2001-01-3585, 2001.

[11] G. Kalghatgi, "Fuel Anti-Knock Quality - Part 1. Engine Studies," Society of Automotive Engineers, no. 2001-01-3584, 2001.

[12] A. D. Yates, A. Swarts and C. Viljoen, "Correlating AutoIgnition Delays and Knock-Limited Spark Advance Data for Different Types of Fuel," SAE International, no. 2005-012083, 2005.

[13] G. Kalghatgi, K. Nakata and K. Mogi, "Octane Appetite Studies in Direct Injection Spark Ignition (DISI) Engines," SAE International, no. 2005-01-0244, 2005.

[14] G. T. Kalghatgi, "Auto-Ignition Quality of Practical Fuels and Implications for Fuel Requirements of Future SI and HCCI Engines," SAE International, no. 2005-01-0239, 2005.

[15] G. Kalghatgi, P. Risberg and H. Angstrom, "A Method of Defining Ignition Quality of Fuels in HCCI Engines," SAE International, no. 2003-01-1816, 2003.

[16] G. Shibata and T. Urushihara, "Auto-Ignition Characteristics of Hydrocarbons and Development of HCCI Fuel Index," SAE International, no. 2007-01-0220, 2007.

[17] I. Truedsson, W. Cannella, B. Johansson and M. Tuner, "Development of New Test Method for Evaluating HCCI Fuel Performance," SAE International, no. 2014-01-2667, 2014.

[18] J. Lacey, K. Kameshwaran, S. Sathasivam, Z. Filipi, W. Cannella and P. Fuentes-Afflick, "Effects of refinery stream gasoline property variation on the auto-ignition quality of a fuel and homogeneous charge compression ignition combustion," International Journal of Engine Research, vol. 18, no. 3, 2016.

[19] J. A. Badra, N. Bokhumseen, N. Mulla, M. Sarathy, A. Farooq, G. Kalghatgi and P. Gaillard, "A methodology to relate octane numbers of binary and ternary n-heptane, iso-octane and toluene mixtures with simulated ignition delay times," Fuel, pp. 458-469, 2015.

[20] E. Singh, J. Badra, M. Mehl and S. M. Sarathy, "Chemical Kinetic Insights into the Octane Number and Octane Sensitivity of Gasoline Surrogate Mixtures," Energy \& Fuels, vol. 31, pp. 1945-1960, 2017.

[21] A. Swarts, A. Yates, C. Viljoen and R. Coetzer, "Standard Knock Intensity Revisited: Atypical Burn Rate Characteristics Identified in the CFR Octane Rating Engine," SAE International, no. 2004-01-1850, 2004.

[22] V. Arrigoni, G. M. Cornetti, G. Spallanzani, F. Calvi and A. Tontodonati, "High Speed Knock in S.I. Engines," Society of Automotive Engineers, no. 741056, 1974.

[23] A. Swarts, A. Yates, C. Viljoen and R. Coetzer, "A Further Study of Inconsistencies between Autoignition and Knock Intensity in the CFR Octane Rating Engine," $S A E$ International, no. 2005-01-2081, 2005.

[24] A. Swarts and A. Yates, "Insights into the Role of Autoignition during the Octane Rating," SAE International, no. 2007-010008, 2007.

[25] Y. B. Zeldovich, "Regime Classification of an Exothermic Reaction with Nonuniform Initial Conditions," Combustion and Flame, vol. 39, pp. 211-214, 1980.

[26] W. Leppard, "A Comparison of Olefin and Paraffin Autoignition chemistries: A Motored-Engine Study," SAE International, no. 892081, 1989.

[27] C. Westbrook, W. Pitz and W. Leppard, "The Autoignition Chemistry of Paraffinic Fuels and Pro-Knock and Anti-Knock Additives: A detailed Chemical Kinetic Study," SAE International, no. 912314, 1991.

[28] G. Kalghatgi, I. Algunaibet and K. Morganti, "On Knock Intensity and Superknock in SI Engines," SAE International , no. 2017-01-0689, 2017.

[29] G. Kalghatgi, K. Morganti and I. Algunaibet, "Some Insights on the Stochastic Nature of Knock and the Evolution of Hot Spots in the End-Gas During the Engine Cycle from Experimental Measurements of Knock Onset and Knock Intensity," SAE International, no. 2017-01-2233, 2017. 
[30] J. Ghandhi and K. S. Kim, "A Statistical Description of Knock Intensity and Its Prediction," SAE International, no. 2017-010659, 2017.

[31] A. J. Shahlari and J. B. Ghandhi, "A Comparison of Engine Knock Metrics," SAE International, no. 2012-32-0007, 2012.

[32] A. J. Shahlari and J. Ghandhi, "Pressure-Based Knock Measurement Issues," SAE International, 2017-01-0668.

[33] ASTM D2699 - 15a, Designation: 237/87, "Standard test method for research octane number of spark-ignition engine fuel"., 2015.

[34] ASTM D2700-16, Designation 236/87, "Standard test method for motor octane number of spark-ignition engine fuel"., 2016.

[35] J. B. Heywood, Internal Combustion Engine Fundamentals, McGraw Hill, 1988.

[36] S. Choi, C. P. Kolodziej, A. Hoth and T. Wallner, "Development and validation of CFR engine GT-Power model for estimating in-cylinder conditions," SAE International, no. 2018-01-0848, 2018.

[37] D. Blackstock, Fundamentals of physical acoustics, John Wiley \& Sons, 2000

[38] G. Koenig, R. R. Maly, D. Bradley, A. K. Lau and C. G. Sheppard, "Role of Exothermic Centres on Knock Inititiation and Knock Damage," SAE International, no. 902136, 1990.

[39] C. P. Kolodziej and T. Wallner, "Combustion characteristics of various fuels during research octane number testing on an instrumented CFR F1/F2 engine," in Combustion Engines PTNSS, Poznan, 2017.

\section{Contact Information}

Toby Rockstroh, trockstroh@anl.gov

\section{Acknowledgments}

The submitted manuscript has been created in part by UChicago Argonne, LLC, Operator of Argonne National Laboratory (“Argonne”). Argonne, a U.S. Department of Energy Office of Science laboratory, is operated under Contract No. DE-AC0206CH11357. The U.S. Government retains for itself, and others acting on its behalf, a paid-up nonexclusive, irrevocable worldwide license in said article to reproduce, prepare derivative works, distribute copies to the public, and perform publicly and display publicly, by or on behalf of the Government. The Department of Energy will provide public access to these results of federally sponsored research in accordance with the DOE Public Access Plan.

http://energy.gov/downloads/doe-public-access-plan. This work is performed under the auspices of the Office of Energy Efficiency and Renewable Energy, Office of Vehicle Technology, U.S. Department of Energy, under contract number DE-AC02-06CH11357, as part of the Co-Optimization of Fuels \& Engines (Co-Optima).

The authors would like to acknowledge Tim Rutter for assisting with maintenance in and around the engine test facility and for commissioning the intake air boost system. Furthermore, the authors would like to express their gratitude to Alexander Hoth for assisting with the engine tests.

\section{Definitions/Abbreviations}

CFR

ASTM

PRF

RON

MON

KLSA

PRR

KP

TSF

KO

KI

KU

MAPO

CAD

aHRR

aHR

IVC

PSD

KSE

OEM
Cooperative Fuels Research

American Society of Testing and Materials

Primary Reference Fuel

Research Octane Number

Motor Octane Number

Knock Limited Spark Advance

Pressure Rise Rate

Knock Point

Toluene Standardization Fuel

Knock Onset

Knock Intensity

Knock Unit

Maximum Amplitude of Pressure Oscillation

Crank Angle Degrees

Apparent Heat Release Rate

Apparent Heat Release

Intake Valve Closing

Power Spectral Density

Knock Spectral Energy

Original Equipment Manufacturer 


\section{Appendix}

Detailed specifications of the CFR engine and control system.

\begin{tabular}{|l|c|}
\hline Combustion chamber & Cast iron, flat “pancake” \\
\hline Compression ratio (-) & Adjustable 4:1 - 18:1 \\
\hline Bore x Stroke (inches) & 3.25 x 4.5 \\
\hline Displacement $\left(\right.$ inch $\left.^{3}\right)$ & 37.33 \\
\hline Intake valve & No shroud, rotating \\
\hline Exhaust valve & Positive 5 CAD \\
\hline Valve overlap & $\begin{array}{c}\text { Top: Chrome-plated, straight-sided } \\
\text { Middle (3): Ferrous, straight-sided } \\
\text { Oil (1): Cast iron, one-piece, slotted } \\
\text { Piston rings }\end{array}$ \\
\hline Fuel system & $\begin{array}{c}\text { Carbureted, adjustable level float } \\
\text { chamber A/F control }\end{array}$ \\
\hline Ignition & Capacitive discharge coil to spark \\
\hline
\end{tabular}

\begin{tabular}{|l|c|}
\hline \multicolumn{2}{|c|}{ Controls } \\
\hline Engine speed & Pulley drive ratio \\
\hline Intake air rel. humidity & Refrigerated intake air conditioner \\
\hline Intake air temperature & Air heater upstream of carburetor \\
\hline $\begin{array}{l}\text { Mixture temperature } \\
\text { (MON only) }\end{array}$ & \begin{tabular}{c} 
Mixture heater downstream of carburetor \\
\hline Compression ratio
\end{tabular} \\
\hline Spark timing & $\begin{array}{c}\text { Worm gear around cylinder to adjust } \\
\text { cylinder head height }\end{array}$ \\
\hline Air-fuel ratio & Mechanically adjusted \\
\hline & Carburetor bowl height \\
\hline Relative air-fuel ratio & Measurements \\
\hline Relative knock & Knockmeter system knock units (KU) \\
\hline
\end{tabular}

